\title{
SUBPRINCIPAL TERMS IN SZEGÖ ESTIMATES
}

\section{Guillemin And K. OKikiolu}

Let $X$ be a compact d-dimensional manifold and $\mathcal{Q}$ a first order positive selfadjoint elliptic pseudodifferential operator (operating on half-densities) whose principal symbol, $q$, satisfies $q(x, \xi)=q(x,-\xi)$, and whose subprincipal symbol is zero. Let $\lambda_{i}, i=1,2, \ldots$ be the eigenvalues of $\mathcal{Q}$ and $f_{i}, i=1,2, \ldots$, the corresponding eigenfunctions, and let $P_{\lambda}$ be the orthogonal projection from the space of half densities onto the space spanned by $f_{i}, \lambda_{i}<\lambda$. The classical "Weyl Theorem", asserts that

$$
\operatorname{trace} P_{\lambda}=\left(\frac{1}{2 \pi}\right)^{d} \int_{q<1} d x d \xi \quad \lambda^{d}+o\left(\lambda^{d}\right)
$$

(the left hand side being the "Weyl counting function" $N(\lambda)$ : the number of $\lambda_{i}$ 's less than $\lambda$.) In [H1] Hörmander showed that the $o\left(\lambda^{d}\right)$ on the right could be improved to an $O\left(\lambda^{d-1}\right)$ and that this error term is optimal (being "best possible", for instance, for $\mathcal{Q}=\left(\Delta_{S^{n}}\right)^{\frac{1}{2}}$.). It turns out, however, that one can frequently replace this $O\left(\lambda^{d-1}\right)$ by an $o\left(\lambda^{d-1}\right)$ : Let

$$
v_{q}=\sum \frac{\partial q}{\partial \xi_{i}} \frac{\partial}{\partial x_{i}}-\frac{\partial q}{\partial x_{i}} \frac{\partial}{\partial \xi_{i}}
$$

be the bicharacteristic vector field on $T^{*} X-0$ associated with $q$. A point $(x, \xi) \in T^{*} X-0$ is periodic if the trajectory of $v_{q}$ through $(x, \xi)$ returns to $(x, \xi)$ after a finite time.

Theorem 1. [DG] If the set of periodic points is of measure zero in $T^{*} X-0$

$$
\operatorname{trace} P_{\lambda}=\left(\frac{1}{2 \pi}\right)^{d} \int_{q<1} d x d \xi \quad \lambda^{d}+o\left(\lambda^{d-1}\right) .
$$

A simple and elegant proof of this result (due to Ivrii) can be found in [H2] $\S$ 29.1. From Ivrii's proof one can also deduce:

Received November 22, 1996.

The first author is supported by NSF grant DMS-890771. The second author is supported by an NSF Postdoctoral Fellowship. 
Theorem 2. Let $A$ be a zeroth order pseudodifferential operator acting on half densities. If the set of periodic points is of measure zero in $T^{*} X-0$, then

(3) $\operatorname{trace} P_{\lambda} A P_{\lambda}=\left(\frac{1}{2 \pi}\right)^{d}\left(\int_{q<1} a(x, \xi) \lambda^{d}+a_{1}(x, \xi) \lambda^{d-1} d x d \xi\right)+o\left(\lambda^{d-1}\right)$,

$a$ being the principal symbol of $A$ and $a_{1}$ its subprincipal symbol.

The "Szego estimates" referred to in the title have to do with the asymptotic behavior of trace $\left(P_{\lambda} A P_{\lambda}\right)^{k}$ as $\lambda$ tends to infinity. By a simple commutation argument of Widom $([\mathrm{H} 2], \S 29.2)$ one can show that

$$
\operatorname{trace}\left(P_{\lambda} A P_{\lambda}\right)^{k}=\operatorname{trace} P_{\lambda} A^{k} P_{\lambda}+o\left(\lambda^{d-1+\varepsilon}\right),
$$

for all $\varepsilon>0$, which in conjunction with (3) implies

$$
\operatorname{trace}\left(P_{\lambda} A P_{\lambda}\right)^{k}=\left(\frac{1}{2 \pi}\right)^{d}\left(\int_{q<1} a(x, \xi)^{k} d x d \xi\right) \lambda^{d}+o\left(\lambda^{d-1+\varepsilon}\right) ;
$$

and recently Laptev and Safarov proved that the $o\left(\lambda^{d-1+\varepsilon}\right)$ in (4) can be replaced by an $O\left(\lambda^{d-1}\right)$ (which gives the same optimal error term for the Szego estimate (3)-(4) as Hörmander obtained for the Weyl estimate.) The purpose at this note is to announce the following result (which can be viewed as the Szego analogue of Theorem 1.)

Theorem 3. Under the hypothesis of Theorem 1

$$
\operatorname{trace}\left(P_{\lambda} A P_{\lambda}\right)^{k}=\operatorname{trace} P_{\lambda} A^{k} P_{\lambda}+c_{k, d-1}(A) \lambda^{d-1}+o\left(\lambda^{d-1}\right)
$$

$c_{k, d-1}(A)$ being equal to

$$
-\frac{d}{4}\left(\frac{1}{2 \pi}\right)^{d+1} \sum_{\substack{r+s=k \\ r, s \geq 1}} \frac{k}{r s} \iint_{-\infty}^{\infty} \frac{\left(a(x, \xi, t)^{r}-a(x, \xi)^{r}\right)\left(a(x, \xi, t)^{s}-a(x, \xi)^{s}\right)}{t^{2}} d t d x d \xi
$$

with $a(x, \xi, t)$ equal to the translate of $a(x, \xi)$ by the bicharacteristic flow, expt $v_{q}$.

Dividing (6) by $k$, summing over $k$, and estimating each summand with respect to an appropriate symbol norm (c.f. "Strong Szegö Theorem" for $P_{\lambda} B P_{\lambda}$ (with $B=I-A$ ).

Theorem 4. Suppose the closed convex hull of the spectrum of B doesn't contain the origin. Then

$$
\log \operatorname{det} P_{\lambda} B P_{\lambda}=\operatorname{trace} P_{\lambda} \log B P_{\lambda}+c_{d-1}(B) \lambda^{d-1}+o\left(\lambda^{d-1}\right)
$$


$c_{d-1}(B)$ being equal to

$$
\frac{d}{4}\left(\frac{1}{2 \pi}\right)^{d+1} \int_{q<1} \int_{-\infty}^{\infty} \frac{(\log b(x, \xi, t)-\log b(x, \xi))^{2}}{t^{2}} d t d x d \xi
$$

with $b(x, \xi, t)=\left(\operatorname{expt} v_{q}\right)^{*} b(x, \xi),(b(x, \xi)$ being the principal symbol of $B$.

(Notice that, under the hypothesis of Theorem $4, \log B$ itself will be a zeroth order pseudodifferential operator; so the first term on the right hand side of (7) can be estimated, modulo $o\left(\lambda^{d-1}\right)$, by (3).)

In the very special case when $\mathcal{Q}$ is a "constant coefficient" operator on the $d$ dimensional torus and $A$ is multiplication by a smooth function, the asymptotic formulas (5), (7) were known, see [Do], [Li], [Ok]. The expressions for $c_{k, d-1}(A)$ and $c_{d-1}(B)$ were given in a different form. The results on the torus guided the discovery of Theorems 3 and 4 .

Remark. From Theorem 3 we can show that if $F$ is a harmonic function on the closed convex hull of the spectrum of $A$ then

$$
\begin{aligned}
& \operatorname{trace} F\left(P_{\lambda} A P_{\lambda}\right)=\operatorname{trace} P_{\lambda} F(A) P_{\lambda}+\frac{d}{2}\left(\frac{1}{2 \pi}\right)^{d} \lambda^{d-1} \times \\
& \int \frac{F((1-\theta) a(x, \xi, t)+\theta a(x, \xi))-[(1-\theta) F(a(x, \xi, t))+\theta F(a(x, \xi))]}{\theta(1-\theta) t^{2}} d \theta d t d x d \xi, \\
& \quad+o\left(\lambda^{d-1}\right)
\end{aligned}
$$

where the integral is over the set where $q<1,-\infty<t<\infty$ and $0<\theta<1$. Compare this formula with Widom [Wi].

What can one say about the asymptotic behavior of trace $\left(P_{\lambda} A P_{\lambda}\right)^{k}$ and $\operatorname{det} P_{\lambda} A P_{\lambda}$ when $A$ doesn't satisfy the hypotheses of Theorem 1, i.e. when the set of periodic points is not of measure zero? Suppose, for instance, that all points are simply periodic of period $T$; i.e. every bicharacteristic returns for the first time to its initial position at time $T$. Replacing $\mathcal{Q}$ by the operator

$$
\mathcal{Q} \log e^{-i T \mathcal{Q}}
$$

one can, without loss of generality, assume that $\exp i T \mathcal{Q}=I$ and hence that

$$
\operatorname{spec} \mathcal{Q}=\left\{\frac{2 \pi n}{T}, n=1,2, \ldots\right\} .
$$

An operator with this property is called a Zoll operator, and for such operators the following very strong Szegö theorem is true: 
Theorem 5. Let $\pi_{k}$ be the orthogonal projection from the space of half densities onto the space spanned by the eigenfunctions of $\mathcal{Q}$ with eigenvalue, $2 \pi k / T$, and let $P_{n}=\pi_{1}+\cdots+\pi_{n}$. Then for every zeroth order pseudodifferential operator $A$

$$
\operatorname{trace}\left(P_{n} A P_{n}\right)^{k} \sim \operatorname{trace} P_{n} A^{k} P_{n}+\sum_{r=d}^{-\infty} c_{k, r}(A) n^{r} \sim c^{\prime}(A) \log n+\sum_{r=d}^{-\infty} c_{k, r}^{\prime}(A) n^{r}
$$

as $n \rightarrow \infty$

Remark. If the symbolic norm of $A$ is sufficiently small, one can divide (11) by $k$ and sum over $k$ to get expression of the form (11) for $\log \operatorname{det} P_{n} B P_{n}$ where $B=I-A$. In particular

$$
\log \operatorname{det} P_{n} B P_{n} \sim \operatorname{trace} P_{n} \log B P_{n}+c_{d-1} n^{d-1}+c_{d-2} n^{d-2}+\cdots
$$

where

$$
c_{d-1}=\frac{d}{4}\left(\frac{1}{2 \pi}\right)^{d+1} \int_{q<1} \int_{-\infty}^{\infty} \frac{(\log b(x, \xi, t)-\log b(x, \xi))^{2}}{t^{2}} d t d x d \xi
$$

$b(x, \xi)$ being the principal symbol of $b$ and $b(x, \xi, t)=\left(\operatorname{expt} v_{q}\right)^{*} b(x, \xi)$; i.e. the coefficient of $n^{d-1}$ in (13) is identical with the coefficient of $\lambda^{d-1}$ in (8). This is also true for the coefficient $c_{k, d-1}$ in (11). For details see [GO2].

We will give a brief sketch of the proof of Theorem 3 .

Let $\rho(\lambda)$ be a Schwartz function which is everywhere positive and whose Fourier transform is supported in the interval, $(-1,1)$. Normalize $\rho$ so that $\int \rho(\lambda) d \lambda=1$, and let $\rho_{\varepsilon}(\lambda)=\rho\left(\frac{\lambda}{\varepsilon}\right)$. Let $P_{\lambda}^{\varepsilon}=\int \rho_{\varepsilon}(\lambda-s) P_{s} d s$. We first prove

Lemma 6. $\left\|P_{\lambda}-P_{\lambda}^{\varepsilon}\right\|_{1} \leq C(\varepsilon+\alpha(\lambda)) \lambda^{d-1}$ where $\alpha(\lambda) \rightarrow 0$ as $\lambda \rightarrow+\infty$.

This estimate enables us to replace trace $\left(P_{\lambda} A\right)^{k}$ by the mollified expression

$$
\operatorname{trace}\left(P_{\lambda}^{\varepsilon} A\right)^{k}
$$

at the expense of introducing an error of order $\varepsilon \lambda^{d-1}+o\left(\lambda^{d-1}\right)$. We next estimate (14). $P_{\lambda}^{\varepsilon}$ can be expressed in terms of the wave operator $e^{i t \mathcal{Q}}$ as

$$
\left(\frac{i}{2 \pi}\right) \int_{-\infty}^{\infty} e^{-i t \lambda} \hat{\rho}_{\varepsilon}(t) \frac{e^{i t \mathcal{Q}}}{t+i 0} d t
$$

From this, we show that $(14)$ is the Fourier transform of trace $e^{i t \mathcal{Q}} G(t)$, i.e.

$$
\operatorname{trace}\left(P_{\lambda}^{\varepsilon} A\right)^{k}=\int_{-\infty}^{\infty} e^{-i t \lambda} \operatorname{trace} e^{i t \mathcal{Q}} G(t) d t
$$


where $G(t)$ equals

$$
\left(\frac{i}{2 \pi}\right)^{k} e^{-i t \mathcal{Q}} \int_{t_{1}+\cdots+t_{k}=t} \hat{\rho}_{\varepsilon}\left(t_{1}\right) \ldots \hat{\rho}_{\varepsilon}\left(t_{k}\right) \frac{e^{i t_{1} \mathcal{Q}} A \ldots e^{i t_{k} \mathcal{Q}} A}{\left(t_{1}+i 0\right) \ldots\left(t_{k}+i 0\right)} d t_{2} \ldots d t_{k} .
$$

When care is taken to ensure that this expression is well defined, it can be seen that $G(t)$ is a zeroth order pseudodifferential operator which varies smoothly in $t$ for $t \neq 0$ and vanishes for $|t|$ large. The asymptotic behavior of (15) can be determined from the behavior of $G(t)$ close to $t=0$. We carry out this analysis in the case $k=2$.

When $k=2, G(t)=G_{0}(t)+G_{1}(t)$, where

$$
\begin{aligned}
& G_{0}(t)=\left(\frac{i}{2 \pi}\right)^{2} \int_{t_{1}+t_{2}=t} \hat{\rho}_{\varepsilon}\left(t_{1}\right) \hat{\rho}_{\varepsilon}\left(t_{2}\right) \frac{A^{2}}{\left(t_{1}+i 0\right)\left(t_{2}+i 0\right)} d t_{2} . \\
& G_{1}(t)=\left(\frac{i}{2 \pi}\right)^{2} \int_{t_{1}+t_{2}=t} \hat{\rho}_{\varepsilon}\left(t_{1}\right) \hat{\rho}_{\varepsilon}\left(t_{2}\right) \frac{e^{-i t_{2} \mathcal{Q}}\left[A, e^{i t_{2} \mathcal{Q}}\right] A}{\left(t_{1}+i 0\right)\left(t_{2}+i 0\right)} d t_{2} .
\end{aligned}
$$

We see that

$$
\int_{-\infty}^{\infty} e^{-i t \lambda} \operatorname{trace} e^{i t \mathcal{Q}} G_{0}(t) d t=\operatorname{trace}\left(P_{\lambda}^{\varepsilon}\right)^{2} A^{2} .
$$

This gives the leading order term in (5) modulo an error of order $\varepsilon \lambda^{d-1}+o\left(\lambda^{d-1}\right)$. Now $G_{1}(t)$ is smooth at $t=0$. The asymptotic behavior of

$$
\int_{-\infty}^{\infty} e^{-i t \lambda} \operatorname{trace} e^{i t \mathcal{Q}} G_{1}(t) d t
$$

can be determined from the following Lemma.

Lemma 7. If $F(t)$ is a zeroth order pseudodifferential operator which varies smoothly in $t$ and vanishes for $|t|$ large, then under the condition of Theorem 1 ,

$$
\int_{-\infty}^{\infty} e^{-i t \lambda} \operatorname{trace} e^{i t \mathcal{Q}} F(t) d t=c(F(0)) \lambda^{d-1}+o\left(\lambda^{d-1}\right)
$$

where for a zeroth order operator $V$ with principal symbol $v$,

$$
c(V)=d\left(\frac{1}{2 \pi}\right)^{d-1} \int_{q<1} v(x, \xi) d x d \xi
$$

Hence, to compute the asymptotic behavior of (19), we just need to evaluate the principal symbol of $G_{1}(0)$ and compute $c\left(G_{1}(0)\right)$. By $(18), G_{1}(0)$ equals

$$
\left(\frac{i}{2 \pi}\right)^{2} \int_{-\infty}^{\infty} \hat{\rho}_{\varepsilon}(-t) \hat{\rho}_{\varepsilon}(t) \frac{e^{-i t \mathcal{Q}}\left[A, e^{i t \mathcal{Q}}\right] A}{(-t+i 0)(t+i 0)} d t
$$


The principal symbol of this expression can be computed by using the Egorov Theorem which we now state.

Theorem 8. For a zeroth order pseudodifferential operator A with principal symbol $a(x, \xi)$, the operator

$$
e^{-i t \mathcal{Q}} A e^{i t \mathcal{Q}}
$$

is a zeroth order pseudodifferential operator with principal symbol

$$
a(x, \xi, t)=\left(\operatorname{expt} v_{q}\right)^{*} a(x, \xi) .
$$

From this, we find that $c\left(G_{1}(0)\right)$ is equal to

$$
-d\left(\frac{1}{2 \pi}\right)^{d+1} \int_{q<1} \int_{-\infty}^{\infty} \frac{\hat{\rho}_{\varepsilon}(-t) \hat{\rho}_{\varepsilon}(t)(a(x, \xi, t)-a(x, \xi)) a(x, \xi)}{(-t+i 0)(t+i 0)} d t d x d \xi
$$

Using the fact that the measure $d x d \xi$ is invariant under the bicharacteristic flow, this is equal to

$$
-d\left(\frac{1}{2 \pi}\right)^{d+1} \int_{q<1} \int_{-\infty}^{\infty} \frac{\hat{\rho}_{\varepsilon}(-t) \hat{\rho}_{\varepsilon}(t)(a(x, \xi)-a(x, \xi,-t)) a(x, \xi,-t)}{(-t+i 0)(t+i 0)} d t d x d \xi
$$

Replacing $t$ by $-t$ in (26), averaging (25) and (26) and letting $\varepsilon \rightarrow 0$ gives

$$
-\frac{d}{2}\left(\frac{1}{2 \pi}\right)^{d+1} \int_{q<1} \int_{-\infty}^{\infty} \frac{(a(x, \xi, t)-a(x, \xi))^{2}}{t^{2}} d t d x d \xi
$$

For the case $k>2$, obtaining the concise formula (6) is more complicated, and involves the Dyson-Hunt-Kac combinatorial formula, (c.f. [GO1]).

We mention that we have obtained an upper bound for (27) as follows.

Lemma 9. Let $A$ be a zeroth order pseudodifferential operator with principal symbol, a, then

$$
\int_{q<1} \int_{-\infty}^{\infty} \frac{(a(x, \xi, t)-a(x, \xi))^{2}}{t^{2}} d t d x d \xi \leq 4 \pi \int_{q<1}|a|^{2}+\frac{\pi^{2}}{6}|\{q, a\}|^{2} d x d \xi .
$$

The proof of Lemma 7 uses the "Ivrii argument" which we referred to above.

Lemma 10. Suppose that $F(t)$ vanishes for $|t| \geq M$. Let $U$ be a conic open subset of $T^{*} X-0$ with the property that for every point $(x, \xi) \in U$ the integral curve of $U_{q}$ with initial point at $(x, \xi)$ doesn't return to $U$ at any time $t \leq M$. Then if $W$ is a pseudodifferential operator with microsupport in $U$, trace $e^{i t \mathcal{Q}} F(t) W$ is a classical conormal distribution of order $d$ in $t$ with microsupport on the set $t=0, \tau>0, \tau$ being the dual cotangent variable to $t$, and (20) holds with $F(t)$ replaced by $F(t) W$. 
We construct a microlocal partition of unity, $W_{i}, i=0,1, \ldots, N \quad W_{i}$ being a zeroth order pseudodifferential operator with microsupport in an open conic set $U_{i}$, such that for "most" $i$ 's, $U_{i}$ satisfies the hypothesis of Lemma 10, and the sum

$$
\sum_{i}^{\prime} \int_{q<1} \sigma\left(W_{i}\right)(x, \xi) d x d \xi
$$

over the remaining $i$ 's is of order $\varepsilon$. To show that these terms make a contribution of order $\varepsilon \lambda^{d-1}$, we show that if $W$ is a zeroth order pseudodifferential operator with positive principal symbol, $w$, then the left hand side of $(20)$ with $F(t)$ replaced by $F(t) W$ is bounded by

$$
C \lambda^{d-1} \int_{q(x, \xi)<1} w(x, \xi) d \xi d x+\circ\left(\lambda^{d-1}\right) .
$$

This completes the proof of Theorem 3 .

\section{References}

[Do] R. Doktorsky, A generalization of the limit theorem of G. Szegö to the multidimensional case, Sibir. Math. Zh. 25 (1984), 20-29.

[DG] J. J. Duistermaat and V. Guillemin, The spectrum of positive elliptic operators and periodic bicharacteristics, Invent. Math. (1975), 39-79.

[GO1] V. Guillemin and K. Okikiolu, Spectral asymptotics of Toeplitz operators on Zoll manifolds, J. Funct. Anal. (to appear).

[GO2] _ Szegö theorems for Zoll operators, Math. Res. Lett. 3 (1996) 1-14.

[H1] L. Hörmander, The spectral function of an elliptic operator, Acta. Math. 121 (1968), $193-218$

[H2] — , The analysis of linear partial differential equations IV, Springer Verlag, Berlin-Heidelberg-New York (1985)

[LS] A. Laptev and Yu. Safarov, Szegö type limit theorems, J. Funct. Anal. 138 (1996), $544-559$.

[Li] I. Linik, The multidimensional analogue of the limit theorem of G. Szegö, Math USSRIzv. 9 (1975), 1323-1332.

[Ok] K. Okikiolu, The analogue of the strong Szegö limit theorem on the torus and the 3-sphere, Ph. D. Diss., Dept. Math. UCLA, Los Angeles, California, (1991).

[Wi] H. Widom, A trace formula for Wiener-Hopf operators, J. Operator Theory 8 (1982), 279-298.

Department of Mathematics, Massachusetts Institute of Technology, Cambridge, MA 02139-4307

E-mail address: vwg@math.mit.edu

Department of Mathematics, University of California, San Diego, La Jolla, CA 92093-0112

Current address: Department of Mathematics, Massachusetts Institute of Technology, Cambridge, MA 02139-4307

E-mail address: okikiolu@math.mit.edu 